\title{
The value of $\mathrm{p} 16^{\mathrm{INK} 4 \mathrm{a}}$ immunostaining for high- grade squamous intraepithelial lesions in human papillomavirus-negative patients
}

\section{Dai Zhang}

Peking University First Hospital

Jie Song

Peking University First Hospital

Xiaosong Zhang

Peking University First Hospital

Hui Bi ( $\square$ bihuicf_09@126.com )

Peking University First Hospital

\section{Research article}

Keywords: P16INK4a immunostaining, High-grade squamous intraepithelial lesions, Human papillomavirus, Cervical cancer, Colposcopically directed cervical biopsy (CDB)

Posted Date: October 15th, 2020

DOI: https://doi.org/10.21203/rs.3.rs-22799/v2

License: (c) (i) This work is licensed under a Creative Commons Attribution 4.0 International License.

Read Full License 


\section{Abstract}

Background : This study aims to evaluate the value of p16 INK4a immunostaining for high-grade squamous intraepithelial lesions in human papillomavirus-negative patients in Beijing, China.

Methods: In this study, we evaluated the value of p16 INK4a immunostaining, as well as cytology and colposcopy, for predicting high-grade squamous intraepithelial lesions (HSIL) in human papillomavirus (HPV)-negative patients by comparing the methods with the haematoxylin and eosin (H\&E) staining pathological diagnosis of HPV-negative patients.

Results: Of 122 patients negative for the high-risk HPV (HR-HPV) subtype, $26(21.3 \%)$ underwent colposcopically directed multiple punch cervical biopsy with a H\&E pathological diagnosis of HSIL and above (HSIL+), 11 patients (9.0\%) had cervical intraepithelial neoplasia (CIN)2, nine patients $(7.4 \%)$ had CIN3, and six patients (4.9\%) had infiltrating carcinoma. Cytology, colposcopy, and p16 INK4a immunostaining had $52.4 \%, 38.5 \%$, and $92.3 \%$ sensitivity, respectively, and $76.2 \%, 94.8 \%$, and $99 \%$ specificity, respectively. The positive predictive value of cytology, colposcopy, and p16 INK4a immunostaining was $31.4 \%, 66.7 \%$, and $96 \%$, respectively, and the negative predictive value was $88.5 \%, 85.1 \%$, and $97.9 \%$, respectively. Compared with H\&E staining, the kappa of cytology, colposcopy, and p16 INK4a immunostaining was $0.327,0.323$, and 0.926 , respectively.

Conclusion: Positive p16 INK4a immunostaining is very strongly consistent with an H\&E diagnosis of CIN2+, and it can be used as an objective detection index for HSIL+ diagnosis of HPV-negative patients with CIN2+.

\section{Background}

Cervical cancer is the third most common malignancy in women worldwide. ${ }^{1}$ Much epidemiological data proves that invasive carcinoma of the cervix is closely related to persistent infection by the high-risk human papillomavirus (HR-HPV) subtype. ${ }^{2}$ Regular cervical cancer screening for women of reproductive age can reduce the morbidity and mortality rates of cervical cancer. Cytological screening is used for cervical cancer screening but faces disadvantages such as low sensitivity and a lack of cytologists. Based on cervical cancer screening of the population in recent years, many studies have found that both combined screening with cytology + HR-HPV subtype detection and preliminary screening with HR-HPV subtype alone enhances the detection rate of the screening. ${ }^{3}$

The management of women with negative HPV detection and the identification of an objective index that can reveal the presence of a lesion and the lesion grade are critical. Recent studies have mainly focused on finding alternative biomarkers of cervical cancer. Furthermore, diagnosis for suspected cervical lesion with negative HR-HPV subtype detection and women with invasive carcinoma of the cervix currently mainly depends on pathological diagnosis, but morphological diagnosis with haematoxylin and eosin (H\&E) staining alone is greatly affected by individual difference and the evaluations of different 
pathologists on lesions of the same grade are inconsistent, particularly when diagnosing cervical intraepithelial neoplasia (CIN).

The tumour suppressor protein $\mathrm{p} 16^{\mathrm{INK} 4 \mathrm{a}}$ is a cyclin-dependent kinase (CDK) inhibitor that is inactivated in many cancers. This inactivation leads to the inactivation of the retinoblastoma protein (Rb). However, in HPV-associated tumours, the HPV E7 protein will combine with Rb, and inactivate it. In this process, p $16^{\text {INK4a }}$ levels increase markedly. ${ }^{4}$ Currently, positive $16^{\text {INK4a }}$ immunostaining can be used as a marker

of high-grade intraepithelial lesions (HSIL). ${ }^{5}$ At present, $\mathrm{p} 16^{1 \mathrm{NK} 4 \mathrm{a}}$ immunostaining is widely used for the precise diagnosis of HPV-related diseases, especially those patients with CIN2. ${ }^{5,6}$ However, there is insufficient research on $\mathrm{p} 16^{\mathrm{INK} 4 \mathrm{a}}$ in HPV-negative patients. Therefore, we conducted this study to evaluate the value of $\mathrm{p} 16^{\mathrm{INK} 4 \mathrm{a}}$ immunostaining for high-grade squamous intraepithelial lesions in human papillomavirus-negative patients

\section{Methods}

\subsection{Subjects}

This was a retrospective study. From January 2014 to December 2014, patients who were negative for the HR-HPV subtype were recruited in this study to evaluate the value of $\mathrm{p} 16^{\mathrm{INK} 4 \mathrm{a}}$ immunostaining for detecting HSIL and above (HSIL+). These patients had undergone colposcopy and colposcopicallydirected multiple punch cervical biopsy at our hospital. In our study, even cases with HPV neg/cytology neg underwent colposcopy because these patients had several clinical symptoms. All biopsy tissues underwent pathological examination. This study was conducted in accordance with the Declaration of Helsinki and approved by the Human Ethics Committee of Peking University First Hospital. All methods were carried out per cervical cancer screening guidelines. All eligible participants provided written, informed consent to be included in this study.

\subsection{Inclusion and exclusion criteria}

Inclusion criteria: (1) patients who were negative for the HR-HPV subtype; (2) older than 18 years of age; (3) patients who have signed informed consent. Exclusion criteria: (1) patients who were pregnant or nursing women; (2) patients whose data were incomplete.

\subsection{Cytological detection}

A liquid-based, thin-layer cytologic preparation was used, and the 2001 Bethesda System (TBS) was used for diagnosis. Tissues were evaluated as within normal limits (WNL), atypical squamous cells of undetermined significance (ASC-US), atypical squamous cells - cannot exclude high-grade squamous intraepithelial lesion (ASC-H), a low-grade squamous intraepithelial lesion (LSIL), HSIL, squamous cell carcinoma (SCC), and atypical glandular cells (AGC).

\subsection{HPV detection}


HPV was tested using the digene Hybrid Capture 2 (HC2) High-Risk HPV DNA Test (QIAGEN, Gaithersburg, MD, USA) with the Rapid Capture System (QIAGEN), which is based on signal amplification using RNA probes to target the entire HR-HPV genome. ${ }^{8}$ All steps were performed according to the manufacturer's protocols. Briefly, cervical brush samples collected in preserve cytological solution underwent denaturation, hybridisation, capture, and amplification of chemiluminescent signal detection. We also used the HybriMax HPV blot, which captures 21 HPV genotypes: namely six low-risk types (HPV 6, 11, 42, 43, 44, and CP8304) and 15 HR types (HPV 16, 18, 31, 33, 35, 39, 45, 51, 52, 53, 56, 58, 59, 66, and 68) that are common in the Chinese population. ${ }^{9}$ The HC2 test was used for 43 patients, and the HPV blot was used for 74 patients.

\subsection{Colposcopy}

Patients with cytological LSIL+ and all patients with AGC underwent colposcopy. Patients with no cytological abnormality or with HPV-negative ASC-US, but with suspected clinical symptoms ( such as contact bleeding, irregular vaginal bleeding, increased vaginal discharge, etc. ) of cervical cancer, also underwent colposcopy. Colposcopy was carried out per standard procedures. We carried out colposcopically-directed multiple punch cervical biopsy for the most abnormal part of the suspected lesion. Cervical four-quadrant randomised biopsy and endocervical curettage were used when the colposcopy was unsatisfactory.

\subsection{Pathological diagnosis of cervical biopsy samples}

We used a three-level classification method for the pathological H\&E-stained sections of CIN1, CIN2, and CIN3. CIN1 was considered LSIL; CIN2 and CIN3 were classified as HSIL.

\subsection{Detection of $\mathrm{p} 16^{\mathrm{INK} 4 \mathrm{a}}$ protein and evaluation of positive immunostaining results The} immunohistochemical method was adopted for detecting p16 ${ }^{\text {INK } 4 a}$. Paraffin sections of cervical tissue

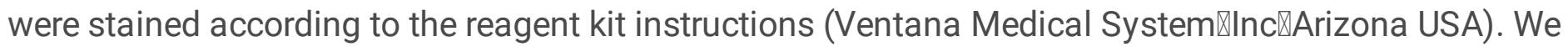
used 1:100 dilution of the primary antibody (mouse anti-human p16 ${ }^{\text {INK4a }}$ monoclonal antibody; clone number E6H4, USA). The primary antibody was replaced with phosphate buffer solution (PBS) to construct the negative control; known p16 ${ }^{\text {INK4a }}$-positive pancreas sections were used as the positive control.

Cells with positive $\mathrm{p} 16^{\mathrm{INK} 4 \mathrm{a}}$ immunostaining had brownish-yellow nuclei and cytoplasm. We determined the staining grade according to the percentage of p16 ${ }^{\mathrm{INK} 4 \mathrm{a}}$-positive cells: positive, epithelial diffuse layer staining; focal positive, focal, discontinuous positive staining; negative, no obvious staining.

\subsection{Cervical loop electrosurgical excision procedure (LEEP)}

For patients with H\&E pathological diagnosis of CIN2 and above (CIN2+), cervical LEEP was carried out in the next menstrual cycle, and the obtained sample was once again pathologically diagnosed. 


\subsection{Statistical analysis}

We used the software program SPSS 13.0 (SPSS, Chicago, IL, USA) to conduct the statistical analysis. The continuous variables of normal distribution were expressed as mean \pm standard deviation, the continuous variables of non-normal distribution were expressed as a median (interquartile range[IQR]), and the categorical variables were expressed as a frequency (percentage[\%]). For two comparisons, each value was compared by t-test. For multiple comparisons, each value was compared by one way ANOVA following Dunnett test when each datum conformed to a normal distribution, while the non-normally distributed continuous data were compared using non-parametric tests. The chi-square test tested the counting data. A value of $\mathrm{P}<0.05$ was considered statistically significant.

\section{Results}

\subsection{The general characteristics}

A total of $122 \mathrm{HR}-\mathrm{HPV}$-negative patients were included in this study. The age of these participants ranged from 19 years to 77 years old. The average age was $42.45 \pm 11.33$ years. Table 1 lists the details.

\subsection{Cervical biopsy pathological results}

Of the 122 patients, 76 patients (62.3\%) had the pathological result of inflammation, 20 patients (16.4\%) had CIN1, 11 patients $(9.0 \%)$ had CIN2, nine patients $(7.4 \%)$ had CIN3, and six patients $(4.9 \%)$ had SCC. In total, 26 patients $(21.3 \%)$ were diagnosed with CIN2+.

\subsection{Value of cytology for detecting HSIL}

We divided the cytology screening results into low-grade abnormality (WNL, ASC-US, LSIL) and high-grade abnormality (ASC-H, HSIL, AGC). Of the 26 patients with CIN2+, 12 patients (46.2\%) were in the low-grade abnormality group, and 14 patients (53.8\%) were in the high-grade abnormality group. CIN2+ detection between the cytology and cervical H\&E pathological results was statistically significantly different (Table 2). The $\mathrm{k}$ value for high-grade abnormality for the cytology and pathological diagnosis of CIN2+ was $0.327\left(\chi^{2}=13.173, P=0.001\right)$.

\subsection{Value of colposcopy for detecting HSIL}

Of the $122 \mathrm{HR}$-HPV-negative patients who underwent colposcopy, 62 patients (50.8\%) were diagnosed as WNL, 45 (36.9\%) were diagnosed as LSIL, 11 (9.0\%) were diagnosed as HSIL, and four (3.3\%) were diagnosed with infiltrating carcinoma. We divided the colposcopy results into low-grade abnormality (WNL and LSIL) and high-grade abnormality (HSIL and infiltrating carcinoma). Eighteen patients (14.8\%) had a high-grade abnormality. There was a statistical difference for CIN2+ detection by colposcopy and H\&E pathological results (Table 3 ). The $\mathrm{k}$ value of the high-grade abnormality for the colposcopy and pathological diagnosis of CIN2+ was $0.323\left(\chi^{2}=13.164, P=0.001\right)$. 


\subsection{Value of p16 ${ }^{\text {INK4a }}$ immunostaining for detecting HSIL}

Of the $122 \mathrm{HR}-\mathrm{HPV}$-negative patients that underwent p16 ${ }^{\text {INK4a }}$ immunostaining, 25 (20.5\%) had positive staining, 20 (16.4\%) had focal positive staining, and 77 (63.1\%) had negative staining. We divided the p16 ${ }^{\text {INK4a }}$ immunostaining results into low-grade abnormality (negative and focal positive staining) and high-grade abnormality (positive staining). There was a statistical difference for CIN2+ detection by p16 ${ }^{\text {INK4a }}$ immunostaining and H\&E pathological results (Table 4). The kappa of the positive p16 $6^{\text {INK4a }}$ immunostaining and pathological diagnosis of CIN2+ was $0.926\left(X^{2}=104.59, P=0.000\right)$.

\subsection{Lower Anogenital Squamous Terminology (LAST) project HSIL diagnosis of cervical biopsy tissue}

In LAST, the diagnostic terminology for different parts of the anus and reproductive tract, and the pathological diagnosis of cervical tissue are consistent with TBS terminology ${ }^{10-11}$. In this study, classification based on cytology, colposcopy, and H\&E pathology detected 26 cases of HSIL+ (CIN2+) (Figure 1); based on LAST, there were 24 cases of HSIL+. The differences were the two cases that were focally positive for $\mathrm{p} 16^{\mathrm{INK} 4 \mathrm{a}}$. After cervical LEEP, one case was diagnosed as CIN1, i.e. LSIL; another was diagnosed as CIN2, i.e. HSIL. The 2 high grade lesions were not considered in the LAST.

\subsection{Pathological results of CIN2+ after cervical LEEP}

All 26 patients with H\&E pathological diagnosis of CIN2+ underwent cervical LEEP, of which 24 had positive $\mathrm{p} 16^{\mathrm{INK} 4 \mathrm{a}}$ immunostaining, and two patients had focal positive $\mathrm{p} 16^{\mathrm{INK} 4 \mathrm{a}}$ immunostaining. The latter two patients underwent cervical LEEP, and returned the pathological result of CIN1 and CIN2, respectively. Of the total 122 patients included in the study, 25 were p $16^{\text {INK4a }}$-positive: one patient with punch biopsy findings of CIN1 is currently under follow-up; the remaining 24 underwent cervical LEEP, and the LEEP specimen pathological results were CIN1 (three patients), CIN2 (nine patients), CIN3 (six patients), and infiltrating carcinoma (six patients). There was a statistically significant difference between the punch biopsy pathological results under colposcopy and the pathological results after cervical LEEP $\left(\chi^{2}=31.704, P=0.000\right)$ (Table 5$)$. The positive predictive value (PPV) of positive p16 INK4a expression for pathological HSIL+ after cervical LEEP was $87.5 \%$.

\subsection{Comparison of detection methods for cervical precancerous lesions}

In summary, $\mathrm{H} \& \mathrm{E}$ staining was the gold standard for detecting cervical precancerous lesions. We evaluated cytology, colposcopy, and p $16^{\text {INK4a }}$ immunostaining for detecting cervical HSIL in cervical HPVnegative patients (Table 6). The sensitivity, specificity, PPV, and negative predictive value (NPV) of p $16^{\text {INK4a }}$ immunostaining were all $>90 \%$, which was significantly better than that of cytology and colposcopy. Including focally positive $\mathrm{p} 16^{\mathrm{INK} 4 \mathrm{a}}$ immunostaining in the detection of cervical precancerous lesions increased the sensitivity and NPV to $100 \%$, but greatly reduced the specificity and PPV, meaning it is not the best choice. 


\section{Discussion}

Numerous epidemiologic studies have proven that persistent HR-HPV infection is the main causative factor of HSIL and infiltrating carcinoma. HR-HPV infection can be detected in almost all patients with precancerous lesion of the cervix and invasive carcinoma of the cervix, and there would be positive clinical detection of an HR-HPV subtype. However, a certain proportion of patients with precancerous lesion of the cervix and invasive carcinoma of the cervix may have negative HR-HPV subtype detection, and the management of such patients should be investigated further in particular. At present, HR-HPV detection enhances screening sensitivity when it is used as an objective index for cervical cancer screening (Early screening or combined cytological screening). 2, 12-13 However, there is currently a lack of more sensitive and objective screening diagnosis indexes for HR-HPV-negative women.

Previous study have found that the reproducibility of H\&E staining morphological diagnoses by pathologists is poor and that the consistency of CIN2 diagnosis is $<50 \% .{ }^{14}$ The reproducibility of CIN2+ morphological diagnosis in the ASCUS/LSIL Triage Study for Cervical Cancer (ALTS) was only $43 \% .{ }^{15}$ In another study, two pathologists agreed with $84 \%$ and $81 \%$, respectively, for CIN3 diagnosis; for CIN2, the agreement was $13 \%$ and $31 \%$, respectively. ${ }^{16}$ There are vast differences among pathologists for $\mathrm{H} \& \mathrm{E}$ staining diagnosis of CIN2, and there are many false positive or false negative results. Recent studies have suggested that auxiliary use of immunohistochemical staining may aid the accuracy of CIN2 diagnosis.

The $16^{\text {INK4a }}$ protein can compete with cyclin D1 to bind with CDK4, inhibiting CDK4 activity. The p16 ${ }^{\text {INK4a }} / \mathrm{CDK}$-cyclin D/Rb complex is the key factor when cells exit the G1 phase of the cell cycle and enter the $S$ phase. Any gene abnormality in this molecular chain may result in the loss of control of molecular action on the cell cycle. The E7 protein expressed by HR-HPV interferes with the normal function of the $R B$ gene. E7 binds with phosphorylated $\mathrm{Rb}(\mathrm{pRb})$, inactivating the function of the $R B$ gene, eliminating the negative feedback inhibition of $\mathrm{pRb}$ on $\mathrm{p} 16^{\mathrm{INK} 4 \mathrm{a}}$ protein expression, and results in p16 ${ }^{\text {INK4a }}$ overexpression. ${ }^{4}$ This leads to disorder of the cell cycle of cervical epithelial cells, resulting in the characteristic of immortality and the initiation of a series of carcinogenesis processes. ${ }^{17-20}$

Li et al. from our department carried out $\mathrm{p} 16^{\mathrm{INK} 4 \mathrm{a}}$ and Ki-67 immunostaining on the pathological sections of patients with $\mathrm{CIN}$ who were aged $<35$ years. These researchers found that p16 $16^{\mathrm{NK} 4 \mathrm{a}}$ and Ki-67 immunostaining had very good consistency with CIN grading. ${ }^{21}$ Galgano et al. found that sensitivity was $86.7 \%$ and specificity was $82.8 \%$ for CIN2+ and that $16^{1 \mathrm{NK} 4 \mathrm{a}}$ immunostaining is a useful and reliable diagnostic adjunct for distinguishing biopsies with and without CIN2+. ${ }^{22}$ Bergeron et al. found that

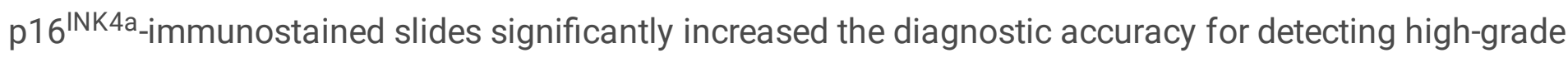
$\mathrm{CIN}$ as compared with H\&E slides and that the reproducibility of $\mathrm{p} 16^{\mathrm{INK} 4 \mathrm{a}}$ immunostaining interpretation was excellent ${ }^{23}$. Other investigators suggested that the conjunctive use of H\&E morphology with p16 ${ }^{\text {INK4a }}$ immunostaining improved inter-observer agreement of the CIN2+ diagnosis. ${ }^{24}$ The LAST Project working group of the College of American Pathologists and ASCCP proposed that the addition of 
p16 ${ }^{\text {INK4a }}$ immunostaining in some cases may provide a more reliable and consistent pathological interpretation. ${ }^{10,25}$ However, can $\mathrm{p} 16^{\mathrm{INK} 4 \mathrm{a}}$ be used as a molecular biological substitute for pathological HSIL of the cervical tissues in HR-HPV-negative patients? Zhang et al. found that diffuse p16 immunostaining is the hallmark of HSIL because it correlates $100 \%$ with CIN2 and CIN3 lesions between initial biopsy and cervical LEEP specimens, whatever the HPV status. ${ }^{26}$ Solano et al. found that p $16^{\text {INK4a }}$ immunostaining had more diagnostic benefits, where their retrospective study of 596 patients revealed HSIL/CIN2-3 not found in the initial H\&E staining. ${ }^{27}$

There was very strong consistency between positive p16 $16^{\text {INK4a }}$ immunostaining and H\&E staining pathological diagnosis in our study (kappa $=0.926)$. The PPV and NPV of high-grade lesion diagnosis was $92.3 \%$ and $97.9 \%$, respectively. Almost $100 \%$ of the cervical HSIL or infiltrating carcinoma could be excluded for patients with negative p $16^{\text {INK4a }}$ immunostaining. For the follow-up of the pathological results, we found that the PPV of p16 ${ }^{\text {INK4a }}$-positive staining for pathological HSIL+ after cervical LEEP was $87.5 \%$, which is higher than that reported by Clinton et al., who found that HSIL detection increased from $48 \%$ to $76 \%(P<0.05)$ after the wide clinical application of p16 ${ }^{\text {INK4a }}$ immunostaining. ${ }^{11}$ Besides, in our study, two patients with H\&E-diagnosed CIN2 had focal positive p16 INK4a immunostaining. The diagnoses of these two patients would be classified as LSIL according to the recommendations of the 2012 LAST guidelines, and follow-up may be conducted for management. The two patients also underwent cervical LEEP in the next menstrual cycle after cervical biopsy, and the results were CIN1 and CIN2, respectively.

Limitations. This study had several limitations. Firstly, this trial was not a randomised controlled trial. Secondly, this study was only a single-centre trial and these 122 HPV negative cases was limited which could not represent the whole patents in our country. Another multiple center trial with large sample size was still needed in the future. Thirdly, diagnostic cervical LEEP can be conducted for elderly women, women with persistent CIN2 for $>2$ years, or patients with other risk factors. As there were only two such patients in this study, more cases should be gathered in the future for an in-depth study to facilitate suggestions for suitable management. Fourthly, it will be better if patients obtained a double negative using both the methods employed to confirm negativity, but as a retrospective study, our patients only received the HPV DNA assays in our hospital which should be further research. Besides, although included samples were HPV negative that the methods can not rule out infection with a genotype not included within the assays used.

Conclusion: Immunostaining for p16 INK4a can be used as a molecular biological substitute for evaluating pathological HSIL of cervical tissue in HR-HPV-negative patients and can be used to aid HSIL detection.

\section{List Of Abbreviations}

HSIL: high-grade squamous intraepithelial lesions

HPV: human papillomavirus 
H\&E: haematoxylin and eosin

HR-HPV: high-risk HPV

CIN: cervical intraepithelial neoplasia

CDB: Colposcopically directed cervical biopsy

H\&E:haematoxylin and eosin

CDK:cyclin-dependent kinase

HSIL:high-grade intraepithelial lesions

TBS:Bethesda System

WNL:within normal limits

ASC-US: atypical squamous cells of undetermined significance

ASC-H:atypical squamous cells - cannot exclude high

LSIL: low-grade squamous intraepithelial lesion

SCC: squamous cell carcinoma

AGC: atypical glandular cells

HC2: Hybrid Capture 2

PBS:phosphate buffer solution)

LEEP: loop electrosurgical excision procedure

IQR: interquartile range

ASCCP: American Society for Colposcopy and Cervical Pathology

PPV: positive predictive value

\section{Declarations}

\section{Ethics approval and consent to participate}

This study was conducted in accordance with the Declaration of Helsinki and approved by the ethics committee of Peking University First Hospital. 


\section{Consent for publication}

Not applicable.

\section{Availability of data and materials}

All data generated or analyzed during this study are included in this published article

\section{Competing interests}

All of the authors had no any personal, financial, commercial, or academic conflicts of interest separately.

\section{Funding}

Not applicable.

\section{Authors' contributions}

ZD and SJ conceived of the study, and ZXS participated in its design and coordination and HB helped to draft the manuscript. All authors read and approved the final manuscript.

\section{Acknowledgements}

Not applicable

\section{References}

1. Ferlay J. et al. GLOBOCAN 2012 v1.0, Cancer Incidence and Mortality Worldwide: IARC Cancer Base No. 11. International Agency for Research on Cancer http://www.globocan.iarc.fr (2013).

2. Arbyn M. et al. Chapter 9: Clinical applications of HPV testing: a summary of meta-analyses. Vaccine 31, S3/78-89 (2006). 
3. Li P. et al. Prognostic value of HPV DNA status in cervical cancer before treatment: a systematic review and meta-analysis. Oncotarget doi: 10.18632/oncotarget.18558. PubMed PMID: 28644141 (2017).

4. Yah L., Michael A. \&amp; Nidhol S. Transcriptional repression of the D-type cyclin-dependent kinase inhibitor P16 by the retinoblastoma susceptibility gene product pRb. Cancer Res. 54, 6078-6082 (1994).

5. Klaes R. et al. Overexpression of p16(INK4A) as a specific marker for dysplastic and neoplastic epithelial cells of the cervix uteri. Int. J. Cancer 92, 276-284 (2001).

6. Sano T, Oyama T, Kashiwabara K, Fukuda T, Nakajima T. Expression status of p16 protein is associated with human papillomavirus oncogenic potential in cervical and genital lesions. Am J Pathol 153ه17411748ه1998凶.

7. Solomon D, et al. The 2001 Bethesda System: terminology for reporting results of cervical cytology. JAMA. 287 (16)/ 2114-9(2002) .

8. Huijsmans CJ, et al. HPV Prevalence in the Dutch cervical cancer screening population (DuSC study): HPV testing using automated HC2, cobas and Aptima workflows. BMC Cancer. 16 (1):922(2016) .

9. Singh S, et al. Distribution of HPV genotypes in Shanghai women. Int J Clin Exp Pathol. 8 (9):11901-8 (2015).

10. Darragh TM, et al. The Lower Anogenital Squamous Terminology Standardization Project for HPVAssociated Lesions: background and consensus recommendations from the College of American Pathologists and the American Society for Colposcopy and Cervical Pathology. J Low Genit Tract Dis. 16:205-242. [PubMed: 22820980](2012) .

11. Tomas Nuño, Francisco García. The LAST Project and its Implications for Clinical Care. Obstet Gynecol Clin North Am. 2013, 40(2): 225-233. doi: 10.1016/j.ogc.2013.02.008.

11. Junyong $\mathrm{H}$, Lixia $\mathrm{H}$. Knowledge of HPV and acceptability of HPV vaccine among women in western China: a cross-sectional survey[J]. BMC Women's Health, 2018, 18(1):130.

12. Sargent A, Fletcher S, Bray K, et al. Cross-sectional study of HPV testing in self-sampled urine and comparison with matched vaginal and cervical samples in women attending colposcopy for the management of abnormal cervical screening[J]. BMJ Open, 2019, 9(4).:e025388.

13. Clinton LK, et al. The LAST guidelines in clinical practice: implementing recommendations for $p 16$ use. Am J Clin Pathol. 144(6):844-9. doi: 10.1309/AJCPUXLP7XD80QYY(2015).

14. Dalla Palma P, Giorgi Rossi P, Collina G, \&amp; NTCC Pathology Group. The reproducibility of CIN diagnoses among different pathologists: data from histology reviews from a multicenter randomized study. Am J Clin Pathol,132: 125-132 (2009). 
15. Castle PE, et al. The relationship of community biopsy-diagnosed cervical intraepithelial neoplasia grade 2 to the quality control pathology-reviewed diagnoses: an ALTS report. Am J Clin Pathol,127:805815 (2007).

16. Carreon JD, et al. CIN2 is a much less reproducible and less valid diagnosis than CIN3: results from a histological review of population-based cervical samples. Int J Gynecol Pathol , 26:441-446(2007).

17. Zur Hausen H. Papillomaviruses and cancer: from basic studies to clinical application. Nat Rev Cancer 2:342-50(2002).

18. Schiffman M, Wentzensen N. From human papillomavirus to cervical cancer. Obstet Gynecol 116:177-85(2010).

19. Bergeron $\mathrm{C}$, et al. Conjunctive P16INK4AINK4a testing significantly increases accuracy in diagnosing high-grade cervical intraepithelial neoplasia. Am J Clin Pathol 133:395-406(2010).

20. Von Knebel Doeberitz M, Reuschenbach M, Schmidt D, Bergeron C. Biomarkers for cervical cancer screening: the role of P16INK4A(INK4a) to highlight transforming HPV infections. Expert Rev Proteomics 9:149-63(2012).

21. Li Siqi, et al. Expression of P16INK4A in grade 2 cervical intraepithelial neoplasia of young women and significance. Chin J Clin Obstet Gynecol, 14 (6): 528-536(2013).

22. Galgano MT, et al. Using biomarkers as objective standards in the diagnosis of cervical biopsies. Am J Surg Pathol. 34:1077-87. [PubMed: 20661011](2010).

23. Bergeron C, et al. European CINtec Histology Study Group. Conjunctive p16INK4a testing significantly increases accuracy in diagnosing high-grade cervical intraepithelial neoplasia. Am J Clin Pathol. 133:395-406. [PubMed: 20154278](2010).

24. Miriam Reuschenbach, et al. p16INK4a Immunohistochemistry in Cervical Biopsy Specimens A Systematic Review and Meta-Analysis of the Interobserver Agreement. Am J Clin Pathol. 142(12):767772. DOI: 10.1309/AJCP3TPHV4TRIZEK.(2014;).

25. Tomas Nuño, Francisco García. The LAST Project and its Implications for Clinical Care. Obstet Gynecol Clin North Am. 40(2): 225-233. doi: 10.1016/j.ogc.2013.02.008.(2013).

26. Zhang G, Yang B, Abdul-Karim FW. p16 Immunohistochemistry is useful in confirming high-grade squamous intraepithelial lesions (HSIL) in women with negative HPV testing. Int J Gynecol Pathol. 34(2):180-186. doi: 10.1097/PGP.0000000000000112.(2015).

27. Solano FJ, Rush DS, Wilkinson EJ. P16 ${ }^{\text {INK4A }}$ immunohistochemical and histopathologic study of Pap test cases interpreted as HSIL without CIN2-3 identification in subsequent cervical specimens. Int J Gynecol Pathol. 34(3):215-220. doi:0.1097/PGP.0000000000000159.(2015)

Page $12 / 16$ 


\section{Tables}

Table 1

Distribution of patient age.

\begin{tabular}{|cccccc|}
\hline Age (years) $₫ 25$ & $25 \llbracket 34$ & $35 \llbracket 44$ & $45 \llbracket 54$ & $55 \llbracket 64$ & $\geq 65$ \\
\hline Percentage $3.3 \%$ & $22.1 \%$ & $35.2 \%$ & $23.8 \%$ & $12.3 \%$ & $3.3 \%$ \\
$(4 / 122)(27 / 122)(43 / 122)(29 / 122)(15 / 122)(4 / 122)$ \\
\hline
\end{tabular}

Table 2

Comparison of cytology results and cervical biopsy pathological results.

\begin{tabular}{|lllllllll|l|}
\hline \multicolumn{7}{|c|}{ Pathological result InflammationCIN1 CIN2 CIN3 SCC } & Total $\chi^{2}$ & $P$ \\
\cline { 2 - 7 } & $\mathrm{n}$ & $\%$ & $\mathrm{n} \%$ & $\mathrm{n} \%$ & $\mathrm{n} \%$ & $\mathrm{n} \%$ & $\mathrm{n}$ & \\
\hline Cytology results & & & & & & & & \\
\hline WNL & 37 & 78.7 & 612.812 .1 & 12.1 & 24.3 & 47 & \\
\hline ASC-US & 14 & 66.7 & 29.5 & 29.5 & 14.8 & 29.5 & 21 & \\
\hline LSIL & 10 & 52.6 & 631.6210 .515 .3 & 00.0 & 19 & 38.2750 .008 \\
\hline ASC-H & 6 & 46.2 & 430.800 .0 & 323.100 .0 & 13 & \\
\hline HSIL & 5 & 29.4 & 211.8529 .4317 .6211 .817 & \\
\hline AGC & 4 & 80.0 & 00.0 & 120.000 .0 & 00.0 & 5 & \\
\hline
\end{tabular}


Table 3

Comparison of colposcopy results and cervical biopsy pathological results.

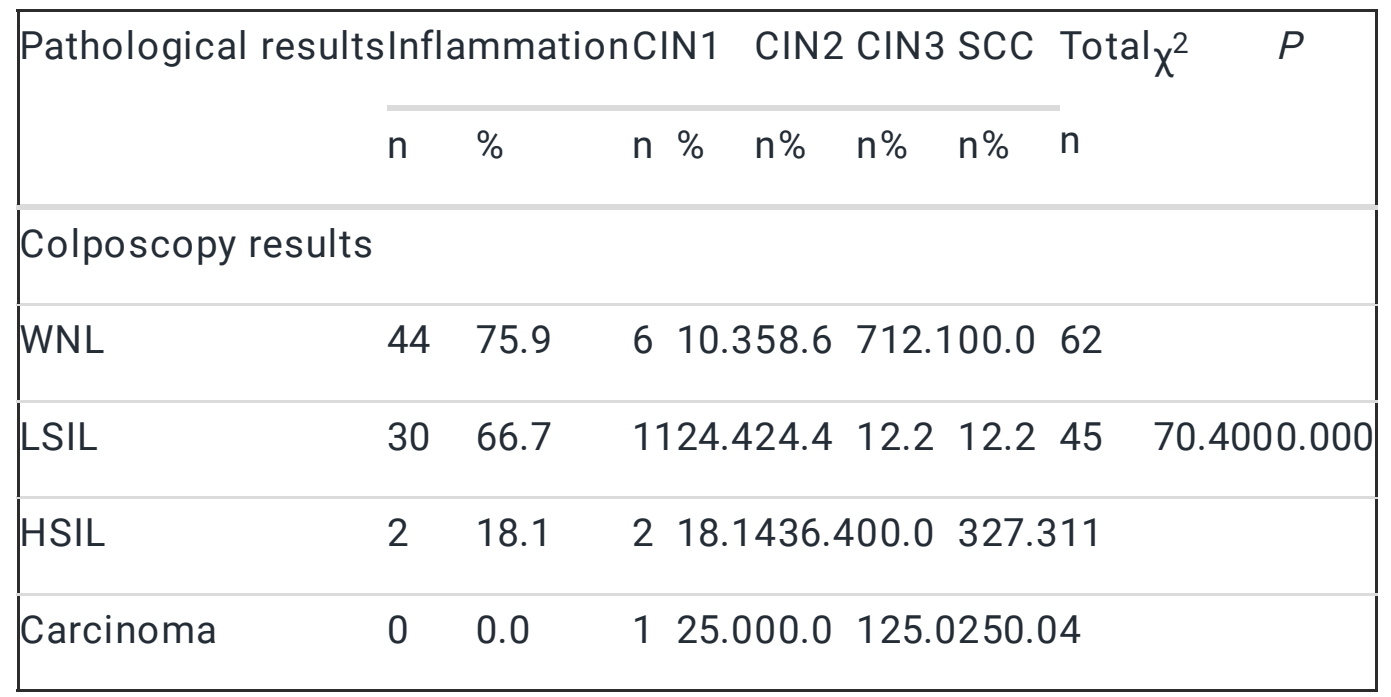

Table 4

Comparison of $\mathrm{p} 16^{\mathrm{INK} 4 a}$ immunostaining results and cervical biopsy pathological results.

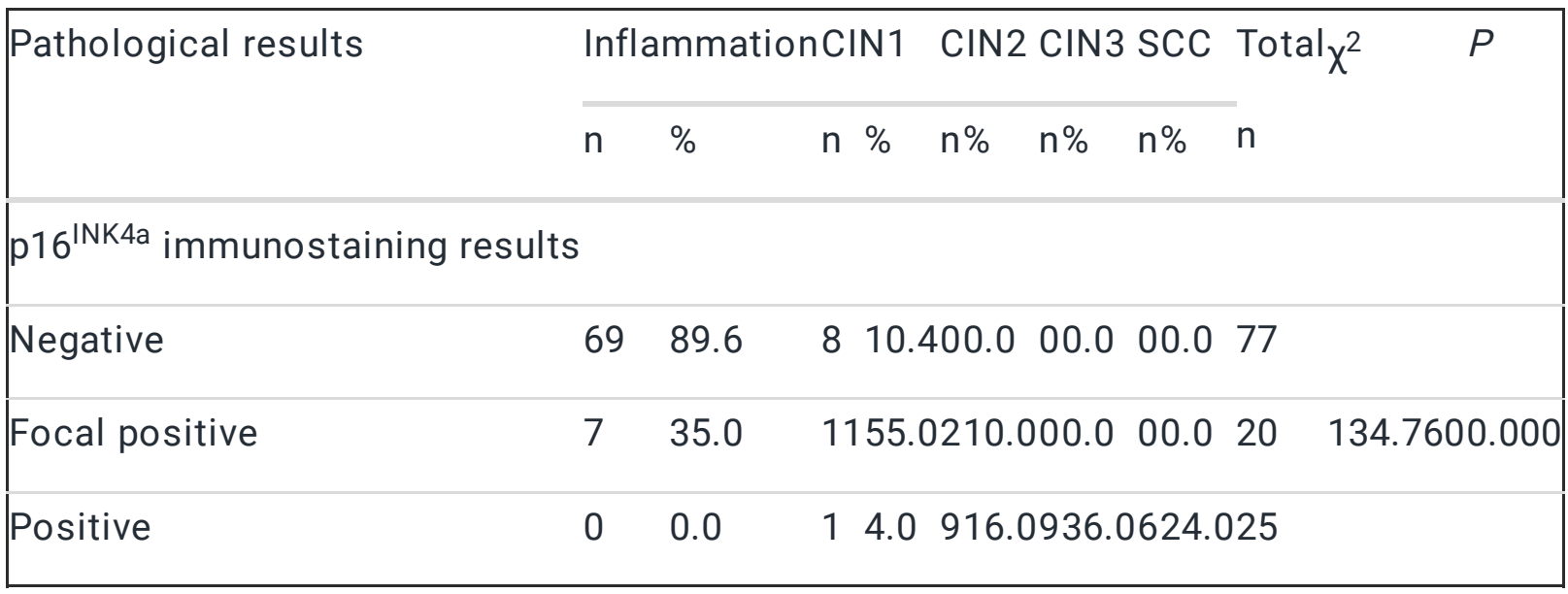


Table 5

Comparison of the pathological results before and after cervical LEEP in p16 ${ }^{\text {INK4a }}$-positive patients.

\begin{tabular}{|c|c|c|c|c|c|}
\hline \multirow{3}{*}{$\begin{array}{l}\text { Colposcopically directed cervical biopsy pathological } \\
\text { results }\end{array}$} & \multicolumn{5}{|c|}{ Pathological results after cervical LEEP } \\
\hline & \multicolumn{2}{|c|}{ CIN1 } & \multicolumn{2}{|c|}{$\begin{array}{r}\text { CIN2 CIN3 Infiltrating } \\
\text { carcinoma }\end{array}$} & \multirow[t]{2}{*}{ Tota } \\
\hline & $\mathrm{n}$ & $\%$ & $\mathrm{n} \% \quad \mathrm{n} \% \mathrm{n}$ & $\%$ & \\
\hline CIN2 & 3 & 33.3 & 3555.6111 .10 & 0.0 & 9 \\
\hline CIN3 & 0 & 0.0 & 444.4555 .60 & 0.0 & 9 \\
\hline Infiltrating carcinoma & 0 & 0.0 & 00.000 .06 & 100.0 & 6 \\
\hline Total & 3 & 12.5 & 5937.5625 .06 & 25.0 & 24 \\
\hline
\end{tabular}

Table 6

Comparison of different methods for detecting cervical precancerous lesions.

\begin{tabular}{|c|c|c|c|c|}
\hline Parameter & Cytolc & Colpo & p16 INK4a immunost & p16 ${ }^{\text {INK4a immunostaining }}$ \\
\hline & & & (positive staining) & $\begin{array}{l}\text { (positive and focal positive } \\
\text { staining) }\end{array}$ \\
\hline Sensitivity & 52.4 & 38.5 & 92.3 & 100 \\
\hline Specificity & 76.2 & 94.8 & 99.0 & 80.2 \\
\hline PPV & 31.4 & 66.7 & 96.0 & 57,8 \\
\hline NPV & 88.5 & 85.1 & 97.9 & 100 \\
\hline $\begin{array}{l}\text { Kappa with } \\
\text { H\&E staining }\end{array}$ & 0.327 & 0.323 & 0.926 & \\
\hline
\end{tabular}

\section{Figures}



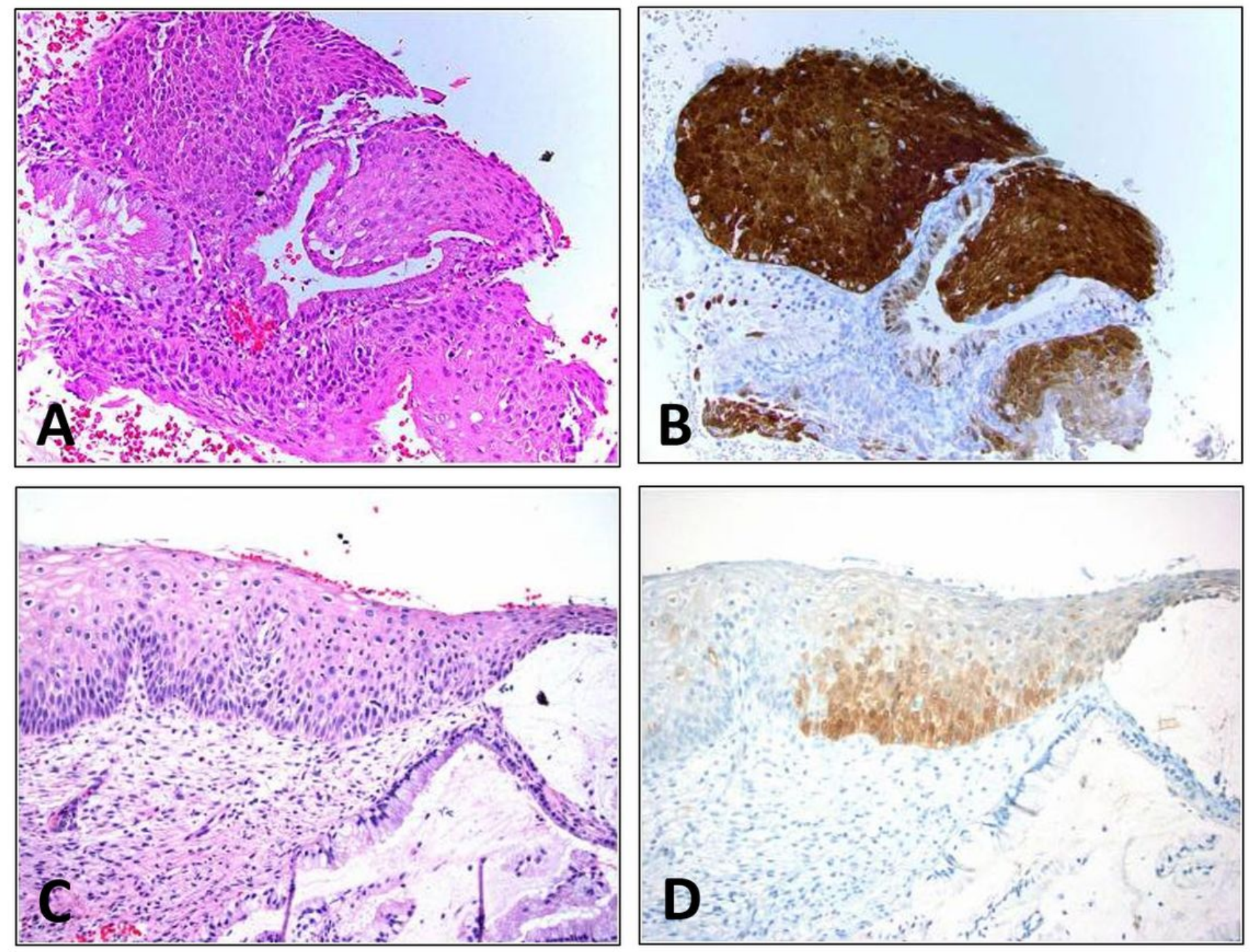

Figure 1

The H\&E staining, cytology, colposcopy, and p16INK4a immunostaining for detecting cervical HSIL in cervical HPV-negative patients. A: The H\&E staining; B: cytology; C: colposcopy; D: p16INK4a immunostaining. 